\title{
A MOLECULAR THEORY OF FILLER \\ REINFORCEMENT BASED UPON THE \\ CONCEPTION OF INTERNAL DEFORMATION \\ (A ROUGH APPROXIMATION OF THE
INTERNAL DEFORMATION)
}

\author{
I \\ Yoshiyasu SATO \\ Faculty of Textile Scionce and Technology, Shinshu University, Ueda \\ and \\ Junji FURUKAwA \\ Department of Industrial Chemistry, Kyoto University, Kyoto \\ (Recived September 10,1962)
}

\begin{abstract}
A molecular theory is presented in this paper which gives a method of analysis for the mechanical properties of the filler-reinforced elastomers, based upon the conception of the internal deformation and the statistical theory of the rubber-like elasticity. ${ }^{2}$ ) By using a suitable model and a few new concepts a proper analysis for such a heterogeneous system is obtained. From the theory the internal mechanism of filler reinforcement is understood. It is made clear that reinforcement consists of three effects: the volume effect, the surface effect and the cavitation effect. From the theory, formulae for the tension, swelling tension, Young's moduli, local stress distribution, strain birefreingence, condition for swelling equilibrium, and so on are derived.
\end{abstract}

\section{INTRODUCTION}

It has long been recognized that rubbery substances and plastic materials are reinforced by incorporation of suitable powdery substances (: reinforced fillers) which improve their mechanical properties such as elastic moduli, hardness, stiffness, resiliens, solvent resistance, plastic viscosity, tensile strength, tear resistance, etc.

Although numerous attempts have been made to clarify and systematize the internal mechanism of filler reinforcement, there is at present no distinct picture of the mechanism, much less a satisfactory theoretical treatment of the phenomena. I)

As factors obstructing the development of a theory for filler reinforcement the following four problems might be mentioned:

(1) Lack of a useful and general method of mechanical analysis for the heterogeneous high elastic materials in which rigid particles are dispersed. 


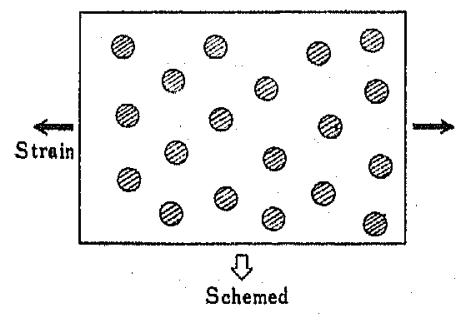

$\sqrt{3}$

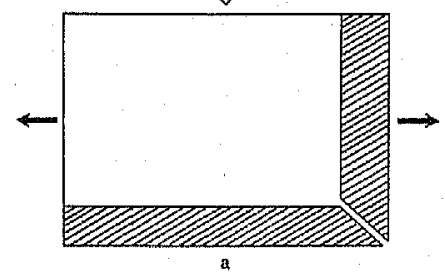

Fig. 1. a: Generally supposed a rough model for heterogeneous materials.

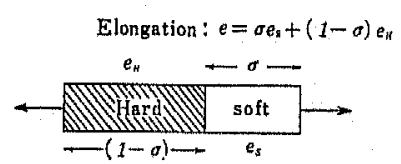

Fig. 1 b:Mullins -Tobins's model.

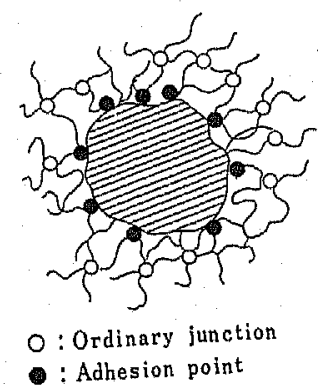

Fig. 2 Adhesion state on surface of particle.
(2) Difficulty in describing analytically the state of adhesion of filler to the chain molecules of the rubber-like substance (refered simply as "rubbery medium" or "medium", henceforth) owing to the variety and complexity of the adhesion. (3) Scanty information and even less analytic representation of the cavitation caused by elongation which is observed characteristically only in filler-reinforced vulcanized rubber.

(4) Very little quantitative knowledge of the effect of the dispersion state of particles on the properties of heterogeneously filled systems. (The term "particle" means a dispersion unit of filler which is not always an ultimate particle in filler powder but is often an aggregation of the ultimate particles).

In order to solve those difficulties, the following treatments are devised. About the difficulty (1), the internal deformatin which is to be distinguished from the apparent deformation in heterogeneous system, is defined by a suitable formula. In regard to the problems (2) and (3), a quantitative representation of the adhesion state which consists of the definition of two idealized adhesion states and the mixing approximation of those is devised. It permits an analysis for the states of adhesion and the cavitation without any assumptions of the molecular mechanism on those phenomena. As to the problem of the dispersion structure (4), an assumption of uniform dispersion of the particles is adopted in this paper.

\section{CONSTRUCTION OF MODEL AND ASSUMPTIONS}

Suppose that $M$ shperical rigid particles with a radius $d$ are uniformly dispersed in a vulcanized rubber-like substance whose volume is $V_{r}$. The volume ratio $X$ of the filler to the rubbery medium and the volume fraction $Y$ of the filler will be given by $X=M(4 \pi / 3) d^{3} / V_{r}$ and $Y=M(4 \pi / 3) d^{3} / V$, respectively, where $V=V_{r}+M(4 \pi / 3) d^{3}$ is the volume of the dispersed system. Since the particles are uniformly dispersed in the medium, the center of each particle is located 
at the lattice point of a face-centered cubic lattice. Let us consider, schematically, a spherical domain concentric to each particle with a radius $D$, such as $D=\{(3 / 4 \pi)(V / M)\}^{1 / 3}$, and regard it as a representation of the dispersed system in the sense of the one-body approximation. This spherical domain is hereafter referred to as the " $D$-sphere", and against this given name the particle is sometimes called the " $d$-sphere".

From the definitions of $X, Y, D$ and the others, next relations are clear

$$
\left.\begin{array}{l}
V=M(4 \pi / 3) D^{8}=V_{r}(1+X), \\
Y=M(4 \pi / 3) d^{3} / V \equiv y^{3} \\
X=M(4 \pi / 3) d^{3} / V_{r}=Y /(1-Y)
\end{array}\right\}
$$

where $y$ stands for $d / D=Y^{1 / 3}$.

$\left(1^{\circ}\right)$ Idealized States of the Adhesion: From the microscopic view there are various interactions between the filler and the chain molecules on the surface of the particles. Some parts of the chain molecules around the particles are fixed on the surface of the filler loosely or tightly according to the type of adhesion. The term "adhesion" in this paper includes both physical attractions and chemical bonds. The actual state of adhesion is approximated by a mixture of two idealized states: the one is the ideal state (ideal state I) in which the medium adheres perfectly to the particles, and the other is the ideal state of perfect non-adhesion (ideal state II) in which the medium and the particles exhibit no interaction.
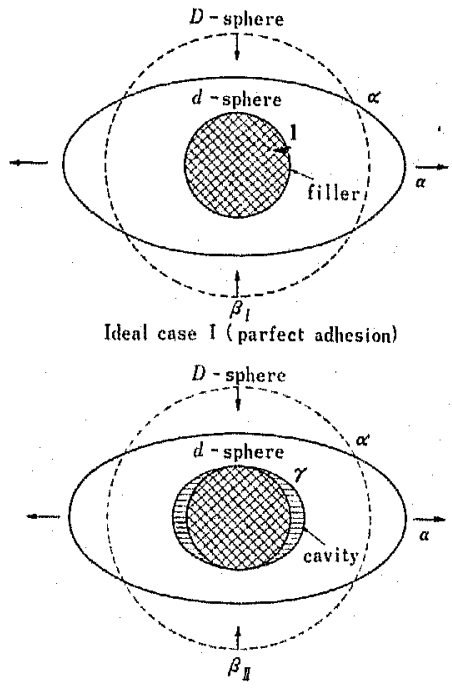

Ideal case II (parfect non - adiesion)

Fig. 3 One-body approximation in terms of D-sphere.
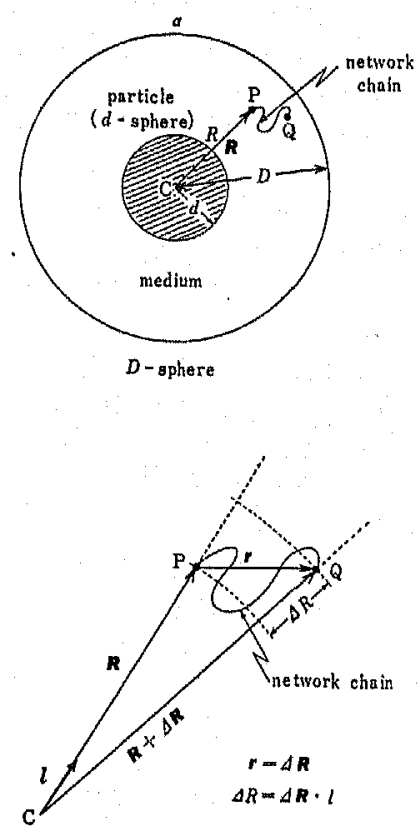

Fig.4 A network chain around point $P$ within rubbery medium in $\mathrm{D}$-sphere. 
$\left(2^{\circ}\right)$ Internal Deformation: In general the uniform external deformation $a$ of the homogeneous materials is a transformation matrix by which any point $\mathrm{P}$ of the material with a position vector $R$ with respect to an arbitrary origin transforms to the point $\mathrm{P}^{\prime}$ with a vector $R^{\prime}$, such as

$$
\boldsymbol{R}^{\prime}=\boldsymbol{\alpha} \boldsymbol{R}
$$

Namely we assume that the internal deformation in the homogeneous materials is equivalent to the external one (:the "requirement of proportionality"). But the internal deformation in the heterogeneous materials must be distinguished from the external or apparent one. Now the internal deformation $\alpha_{R}$ in the present theory is defined by the following formulae, with respect to the center of $D$-sphere taken as the origin

$$
\boldsymbol{R}^{\prime}=\boldsymbol{\alpha}_{R} \boldsymbol{R},
$$

and

$$
\begin{aligned}
\boldsymbol{\alpha}_{R} & =\frac{D R-d}{R D-d} \boldsymbol{a}+\frac{d}{R} \frac{D-R}{D-d} \boldsymbol{\gamma} \\
& =\boldsymbol{\alpha}^{\prime}-(D / R) \boldsymbol{a}^{\prime \prime},
\end{aligned}
$$

and $\boldsymbol{\alpha}^{\prime}, \boldsymbol{\alpha}^{\prime \prime}$ imply that

$$
\boldsymbol{\alpha}^{\prime}=(\boldsymbol{\alpha}-y \gamma) /(1-y), \quad \boldsymbol{\alpha}^{\prime \prime}=\{y /(1-y)\}(\boldsymbol{\alpha}-\boldsymbol{\gamma}),
$$

where $\gamma$ represents the transformation matrix in which the points of the inner surface of the rubbery medium facing the particles transforms to their deformed positions according to the external deformation $a$.

It can make sure easily that $\alpha_{R}$ satisfies the following conditions:

(i) $a_{R}=\alpha$ over the $D$-sphere.

(ii) $a_{R}=\gamma$ over the $d$-sphere.

(iii) $\boldsymbol{\alpha}_{n} \longrightarrow \boldsymbol{a}$ as $Y \longrightarrow 0$.

In addition to the above conditions, the following condition is necessary for the determination of $r$ :

(iv) $r$ minimizes the free energy of the system under the external deformation $\alpha$.

(As a matter of fact, the formula of $\alpha_{R}$ must be determined from the above conditions in opposition to this paper. Although more correct formula for $\alpha_{R}$ is obtained in this way, and more accurate theory is derived based on it, they are not touched upon in this paper).

$\left(3^{\circ}\right)$ Shape and Size of the Cavity: Let us consider the external deformation $\alpha$ of our specimen in simple elongation with the elongation ratio $\alpha_{3}=\alpha$ along the $z$-axis and the contraction ratio $\beta$ along the $x$ and $y$-axes:

$$
\boldsymbol{\alpha}=\left(\alpha_{i} \delta_{i} j\right)=\left(\begin{array}{lll}
\beta & 0 & 0 \\
0 & \beta & 0 \\
0 & 0 & \alpha
\end{array}\right),
$$

where $\delta_{i j}$ is Kronecker's delta

$$
\delta_{i j}= \begin{cases}1, & \text { (if } i=j) \\ 0, & \text { (if } i=j)\end{cases}
$$


Now consider the matrix $\gamma$ under the simple elongation. In the ideal state $I$, the adhesion holds despite of any external deformation of the specimen. While in the ideal state II, the parts of medium contacted with the filler slide over the particle surfaces smoothly or separate themselves from the surface freely according to the magnitude of the elongation ratio $\alpha$, then the cavities are appearing on the both sides of each particle. The shape of the surface of the cavity is considered to resemble closely to an ellipsoid of revolution with minor axes ( $x$ and $y$-axes), $d$, and a major axis (z-axis), $r d,(1 \leq r)$, from the consideration of the axial symmetry. Therefore, the shape of the cavity is assumed to be the ellipsoid of revolution above mentioned, and then $\gamma$ is an unknown function of $\alpha$ which is determined so as to minimize the free energy of deformation of the specimen under a constant elongation $\alpha$.

Then the deformation of the cavity $\gamma$ can be written as

$$
r=\left(\gamma_{i} \delta_{i j}\right)=\left(\begin{array}{lll}
1 & 0 & 0 \\
0 & 1 & 0 \\
0 & 0 & \gamma
\end{array}\right)
$$

To distinguish the quantities for two ideal states I and II, suffixes I and II will henceforth be appended to them respectively, and the quantities with out suffix denote them in the general state. Thus we may now write

$$
r_{\mathrm{I}}=\left(\begin{array}{lll}
1 & 0 & 0 \\
0 & 1 & 0 \\
0 & 0 & 1
\end{array}\right), \quad r_{\mathrm{II}}=\left(\begin{array}{lll}
1 & 0 & 0 \\
0 & 1 & 0 \\
0 & 0 & \gamma
\end{array}\right),
$$

or

$$
r_{\mathrm{I}}=1, \quad r_{\mathrm{II}}=1+\varepsilon
$$

where 1 and $\varepsilon$ are defined as follows:

$$
\mathbf{I}=\left(\delta_{i j}\right)=\left(\begin{array}{ccc}
1 & 0 & 0 \\
0 & 1 & 0 \\
0 & 0 & 1
\end{array}\right), \quad \varepsilon=\left(\begin{array}{ccc}
0 & 0 & 0 \\
0 & 0 & 0 \\
0 & 0 & \gamma-1
\end{array}\right) .
$$

By the comparison between $\gamma_{\mathrm{I}}$ and $\gamma_{\text {II }}$ in Eq. $\left(6^{\prime \prime}\right), \gamma$ in the general state may be represented by

$$
r=1+\zeta \varepsilon, \quad(0 \leq \zeta \leq 1)
$$

where $\zeta$ is a parameter depending on the nature of adhesion between the rubbery medium and the particles such as $\zeta=0$ and $\zeta=1$ correspond to the respective adhesion states of the ideal cases I and II.

$\left(4^{\circ}\right)$ Mixing Approximation and $\mathrm{It}^{\prime} \mathrm{s}$ Application: For the purpose of representing the adhesion state in the general state an approximation of mixing is adopted, so that the general adhesion state is approximated by the one in the mixed system comprising idealized states of perfect adhesion and perfect non-adhesion with a mixing ratio $(1-\zeta): \zeta$, respectively, where $\zeta$ denotes a characteristic parameter corresponding to the adhesion state in question. According to the mixing approximation, the volume $V^{\prime}$, the free energy $F(\alpha)$ and $\gamma$ of the general specimen in the deformed state can be writen as 


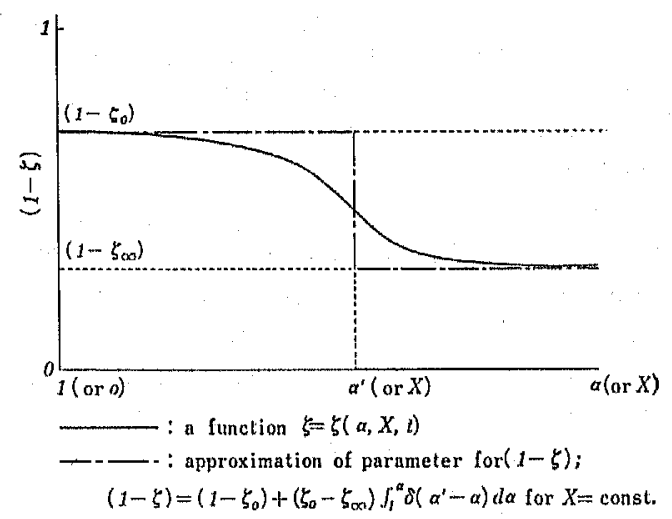

Fig. 5 Schematic representation of change in degree of adhesion $(1-\zeta)$ with respect to $\alpha$ (as $X$ is fixed) or to $X$ (as $a$ is fixed.)

$$
\begin{aligned}
& V^{\prime}=(1-\zeta) V_{\mathrm{I}}{ }^{\prime}+\zeta V_{\mathrm{II}}{ }^{\prime}, \\
& F(\boldsymbol{\alpha})=(1-\zeta) F_{\mathrm{I}}(\boldsymbol{\alpha})+\zeta F_{\mathrm{II}}(\boldsymbol{\alpha})
\end{aligned}
$$

and

$$
\begin{aligned}
r & =(1-\zeta) \gamma_{\mathrm{I}}+\zeta \gamma_{\mathrm{II}} \\
& =1+\zeta \varepsilon .
\end{aligned}
$$

Naturally Eq. (*) is equivalent to the presumption on $\gamma$ in Eq. $\left(6^{\prime \prime \prime}\right)$.

Moreover the surface densities of the network chain adhered on the surface of the particles in the ideal state I, $g_{f}^{(m)}$, and the general state, $g_{f}$, are given as

$$
\begin{aligned}
g_{f} & =(1-\zeta) g_{f}{ }^{(m)}+\zeta \cdot 0 \\
& =g_{f}(m)(1-\zeta),
\end{aligned}
$$

because the surface density in the ideal state $I I$ is 0 . Since the mixing ratio $(1-\zeta)$ plays the role of the reduction ratio from $g_{f}(m)$ to $g_{f},(1-\zeta)$ is called the "degree of adhesion" hereafter. When the volume of rubbery medium $V_{r}$ is kept constant, Eq. (7) becomes

$$
\phi=1+Y(\gamma-1) \zeta
$$

where

$$
\phi=V^{\prime} / V=\alpha \beta^{2} .
$$

$\left(5^{\circ}\right)$ Deformation Theorem, Local Deformation: Since the requirement of proportionality in the ordinary theory of rubber elasticity no longer holds validity for the heterogeneous system, it must be replaced by the deformation theorem of the network chains in our system. Consider a network chain in the $D$-sphere with end-to-end vector $r$. which is connecting a junction at the point $R$ to the other junction at $R+\Delta R$. By using the definition of internal 
deformation in Eq. (3), $\left(4^{\prime}\right)$, the deformation can be derived in the following way:

$$
\begin{aligned}
\Delta R^{\prime} & =\Delta\left(\alpha_{R} R\right) \\
& =\alpha_{R} \Delta R+\left(\Delta \alpha_{R}\right) R \\
& =\alpha_{P} \Delta R,
\end{aligned}
$$

or

$$
r^{\prime}=\alpha_{p} r
$$

where

$$
\begin{aligned}
\boldsymbol{a}_{P}= & \boldsymbol{\alpha}_{R 2}+(D / R) \boldsymbol{\alpha}^{\prime \prime}(l l), \\
& \left(\boldsymbol{\alpha}^{\prime \prime}=\{y /(1-y)\}(\boldsymbol{\alpha}-\boldsymbol{\gamma}),\right.
\end{aligned}
$$

and $r^{\prime}=\Delta R^{\prime}, r=\Delta R$, also $l=R / R$, and $(l l)$ denotes a dyadic with $i j$ components $l_{i} l_{j}$ (Appendix A cf.). As a matter of course the deformation theorem in $\mathrm{Eq}$. (12) becomes the requirement of proportionality in Eq. (2), when the concentration of the filler $Y$ tends to 0 . In the formulae (12), (12a), $\boldsymbol{\alpha}_{l}$, is called the "local deformation" around the point $\mathrm{P}$ defined by $\boldsymbol{R}$.

$\left(6^{\circ}\right)$ Arrangement of Main Assumptions: In the present consideration, the filler-reinforced rubber-like substance is considered as a double network model which is constructed both from the ordinary network chains in the medium with volume density $g_{r}$ and from the adhered network chains over the particle surfaces with surface density $g_{f}=g_{f}(m)(1-\zeta)$.

It is convenient to arrange the assumptions in the theory, about here:

(a) Spherical particles are uniformly dispersed in the rubbery medium.

(b) The movements of the center of each particle uncler the external deformation accords to the requirment of proportionality.

(c) The one-body approximation in terms of $D$-sphere is adopted.

(d) The adhesion state is represented by the mixing approximation.

(e) The shape of the surface of cavity assumes the ellipsoid of revolution.

(f) The volume of rubbery medium is kept constant.

(g) The usual assumptions in the ordinary theory of rubber elasticity are adopted here except the requirement of proportionality: such as

$(\alpha)$ The network chains are Gaussian.

$(\beta)$ The free energy of the system consists of two parts: the part by the sum of the entropy of individual chains and the liquid like interaction energy $U(\phi)$ among the segments ( $U(\phi)$ is a function of $\phi$ only in Eq. (10), and is related with (f)).

\section{ENERGY OF DEFORMATION OF DOUBLE NETWORK SYSTEM}

The free energy of the system per unit initial volume $F(\boldsymbol{\alpha})$ under the external deformation may be devided into two parts: the volume energy $F_{r}(\boldsymbol{\alpha})+U(\phi)$ and the surface energy $F_{f}(\boldsymbol{\alpha})$, then

$$
F(\boldsymbol{\alpha})=F_{r}(\boldsymbol{\alpha})+F_{f}(\boldsymbol{\alpha})+U(\phi) .
$$

Moreover the volume energy consists from the entropy part $F_{r}(\boldsymbol{\alpha})$ and the internal energy $U(\phi)$. The former is induced from the entropy of ordinary 
network chains which are spatially distributed in rubbery medium with volume density $g_{r}$. The surface energy $F_{f}(\alpha)$ is induced from the entropy of adhered network chains whose ends in one side are distributed on the particle surface with surface clensity $g_{f}(m)$ in the ideal state I.

As well known the entropy of a Gaussian chain with end-to-end vector $r$ and $N$ segments of each length $a$ is represented by

$$
\frac{3 k T}{2 N a^{2}} r^{2}
$$

where $k$ is Boltzmann's constant and $T$ is the absolute temparature. When the external deformation proceeds through $1 \rightarrow a$, the above entropy should increases in such a way

$$
\begin{aligned}
\frac{3 k T}{2 N a^{2}} r^{2} & \longrightarrow \frac{3 k T}{2 N a^{2}} \mathbf{r}^{\prime 2} \\
& =\frac{3 k T}{2 N a^{2}}\left(\boldsymbol{\alpha}_{r^{2}} r \cdot \alpha_{r^{n}} r\right)
\end{aligned}
$$

becautse of $r \longrightarrow \boldsymbol{r}^{\prime}=\boldsymbol{\alpha}_{r} \boldsymbol{r}$ according to the deformation theorem in Eq. (12).

$\left(1^{\circ}\right)$ The Volume Energy of Deformation $\Delta F_{r}(\alpha)$ : The entropy part of the volume energy contained in a unit volume about a point $\mathrm{P}$ at the position $R$ is given by

$$
\sum_{N} g_{N} \frac{3 k T}{2 N a^{2}} \int\left(\boldsymbol{\alpha}_{P} r \cdot \boldsymbol{\alpha}_{P} r\right) f(r, N) d r
$$

where the quantity $f(r, N)$ is defined as the probability that one end of a network chain which consists of $N$ segments with end-to-end vector $r$ will be in the volume element $d r$ when the other end is fixed at $R$, and $g_{N}$ is the number of the network chains with $N$ segments per unit volume of rubbery medium, then

$$
g_{r}=\sum_{N} g_{N}
$$

Therefore, $F(\alpha)$ can be obtained by the integration of the entropy in Eq. (15) taken with respect to the medium of all $D$-sphere such as (Appendix B cf.)

$$
\begin{aligned}
F_{r}(\boldsymbol{\alpha}) & =(M / V) \int\left[\sum_{N} g_{N}\left[3 k T /\left(2 N a^{2}\right)\right\} \int\left(\boldsymbol{\alpha}_{p}, \boldsymbol{r} \cdot \boldsymbol{\alpha}_{P} r\right) f(r, N) d r\right] d \boldsymbol{R} \\
& =\{M G /(2 V)\} \int_{(D)} \operatorname{Tr} \cdot\left(\tilde{\boldsymbol{\alpha}}_{P} \boldsymbol{\alpha}_{P}\right) d \boldsymbol{R} \\
& =\frac{G}{2}\left\{\left(1+\frac{y^{2}}{1-y}\right) I_{a a}-2 \frac{y^{2}}{1-y} I_{a r}+y^{2}\left(1+\frac{y^{2}}{1-y}\right) I_{r r}\right\},
\end{aligned}
$$

where $G=g_{r} k T, \int_{(D)} \ldots \ldots \ldots d R$ denotes the volume integral taken with respect to the rubbery medium in each $D$-sphere, $\tilde{a}$ denotes the transpose matrix of $\boldsymbol{a}$, and $I_{A B}$ denotes 


$$
I_{A B}=\operatorname{Tr} .(\mathrm{AB})=\sum_{i, k} A_{i k} B_{i k}
$$

for arbitrary matrices $\mathbf{A}$ and $\mathbf{B}$, then

$$
\begin{aligned}
& I_{a a}=\sum_{i} \alpha_{i}^{2}=2 \beta^{2}+\alpha^{2}, \\
& I_{a r}=\sum_{i} \alpha_{i} \gamma_{i}=2 \beta+\alpha \gamma_{s}, \\
& I_{r r}=\sum_{i} \gamma_{i}^{2}=2+\gamma_{3}, \\
& I_{1}=\operatorname{Tr} . \mathbf{1}=3 .
\end{aligned}
$$

Hence the energy of deformation $\Delta F_{r}(a)$ is defined as the increase in $F_{r}(a)$ through the process of deformation $1 \rightarrow a$, such as

$$
\Delta F_{r}(\boldsymbol{\alpha})=F_{r}(\boldsymbol{\alpha})-F_{r}(\mathbf{1}) \text {. }
$$

When $\gamma_{\mathrm{I}}=1$ and $\gamma_{\mathrm{II}}=\gamma$ are substituted in $\gamma_{3}$ in Eq. (17), we obtain

$$
\Delta F_{r} \mathrm{I}(\alpha)=\frac{G}{2}\left\{\left(1+\frac{y^{2}}{1-y}\right)\left(I_{a \alpha}-I_{1}\right)-2 \frac{y^{2}}{1-y}\left(I_{a}-I_{1}\right)\right.
$$

and

$$
\begin{gathered}
\Delta F_{r \Pi \mathrm{I}}(\boldsymbol{\alpha})=\frac{G}{2}\left\{\left(1+\frac{y^{2}}{1-y}\right)\left(I_{\text {Ia }}-I_{1}\right)-2 \frac{y^{2}}{1-y}\left(I_{a \alpha}-I_{1}\right)\right. \\
\left.\left.+y^{2}\left(1+\frac{y^{2}}{1-y}\right) \cdot I_{r r}-I_{1}\right)\right\} .
\end{gathered}
$$

Using the mixing approximation, the relations $I_{a r}=I_{\alpha}+I_{\alpha \gamma}$ and $I_{r r}=\left(r^{2}-1\right)$, $F_{r}(\boldsymbol{\alpha})$ in the general state can be written as follows:

$$
\begin{aligned}
\Delta F_{r}(\boldsymbol{\alpha})= & (1-\zeta) \Delta F_{r \mathrm{I}}(\boldsymbol{\alpha})+\zeta \Delta F_{r \mathrm{II}}(\boldsymbol{\alpha}) \\
= & \frac{G}{2}\left\{\left(1+\frac{y^{2}}{1-y}\right)\left(I_{\alpha a}-I_{1}\right)-2 \frac{y^{2}}{1-y}\left(I_{a}-I_{1}\right)\right. \\
& \left.-2 \frac{y^{2}}{1-y} \alpha(r-1) \zeta+y^{2}\left(1+\frac{y^{2}}{1-y}\right)\left(r^{2}-1\right) \zeta\right\}
\end{aligned}
$$

$\left(2^{\circ}\right)$ The Surface Energy of Deformation $d F_{f}(\alpha)$ : Similarly the surface energy per unit initial volume of the system in the deformed state $F_{f}(\boldsymbol{\alpha})$ can be calculated by almost the same way as in $F_{r}(\boldsymbol{\alpha})$ except $F_{f \text { II }}(\boldsymbol{\alpha})=0$, because there are not any adhered chains over the surface of all particles in the ideal state II: $g_{f \text { II }}=0$.

In this case the local deformation $\alpha_{P}$ in the ideal state $I$ is reduced to the next form according to Eq. (12a)

$$
\left[\alpha_{P}\right]_{\substack{r=1 \\ R=d}}=1+(a-1)(l l) /(1-y) \text {. }
$$

The surface energy per unit area on the surface of the particles is given by

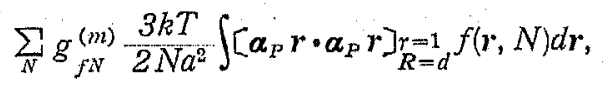

hence $F_{f I}(\alpha)$ is given as the surface integral of above entropy over each 
surface $S$ of all particles in the system: (Appendix cf.)

$$
\begin{aligned}
& F_{f I}(\boldsymbol{\alpha})=(M / V) \int\left[\sum_{N} g_{, N}^{(m)}\left\{3 k T /\left(2 N a^{2}\right)\right\} \int\left[\boldsymbol{\alpha}_{p^{\prime}} \boldsymbol{r} \cdot \boldsymbol{\alpha}_{r^{\prime}} \boldsymbol{r}\right]_{\substack{r=1 \\
R=d l}} f(\boldsymbol{r}, N) d \boldsymbol{r}\right] d S \\
& =\frac{M 4 \pi d^{2}}{2 V} g_{f}^{(m)} k T \frac{1}{3}\left\{3 I_{1}+\frac{2}{1-y}\left(I_{a}-I_{1}\right)+\frac{1}{(1-y)^{2}}\left(I_{a a}-2 I_{a}+I_{1}\right)\right\} \\
& =\frac{g_{j}^{(m)} k T}{2 d} \frac{y^{3}}{(I-y)^{2}}\left\{I_{r a}-2 y I_{a}+\left(3 y^{2}-4 y+2\right) I_{1}\right\},
\end{aligned}
$$

where $g_{f N}(m)$ denotes the surface density of the adhered network chains with $N$ segments in the ideal state I, and hence $\sum_{N} g_{f N}(m)=g_{f}(m)$. As before

$$
\Delta F_{f \mathrm{I}}(\boldsymbol{\alpha})=\frac{g_{f}^{(m)} k T}{2 d} \frac{y^{3}}{(1-y)^{2}}\left\{\left(I_{a a}-I_{1}\right)-2 y\left(I_{a}-I_{1}\right)\right\}
$$

is obtained. From the fact $\Delta F_{f \mathbb{I I}}(\boldsymbol{\alpha}) \equiv 0$, we get

$$
\begin{aligned}
\Delta F_{f}(\boldsymbol{\alpha}) & =(1-\zeta) \Delta F_{f \mathrm{I}}(\boldsymbol{\alpha})+\zeta d F_{f \mathrm{II}}(\boldsymbol{\alpha}) \\
& =\frac{G^{\prime} y^{3}}{2(1-y)^{2}}\left\{\left(I_{u a}-I_{1}\right)-2 y\left(I_{a}-I_{1}\right)\right\},
\end{aligned}
$$

here

$$
G^{\prime}=g_{s}^{(m)}(1-\zeta) k T / d
$$

is a surface modulus which is converted into the volume modulus equivalent to $G=g_{r} h T$.

$\left(3^{\circ}\right)$ The Free Energy of Deformation $\Delta F(\alpha)$ : When $\Delta F_{r}(\alpha)$ in Eq. (19) and $d F_{f}(\boldsymbol{\alpha})$ in $\mathrm{Eq} .\left(24^{\prime}\right)$ are substituted in Eq. (13), the free energy of deformation of the system $\Delta F(\boldsymbol{\alpha})$ can be derived as follows;

$$
\begin{aligned}
\Delta F(\boldsymbol{\alpha})= & (1 / 2)\left\{A_{\zeta}\left(I_{a \alpha}-I_{1}\right)-2 B_{\zeta}\left(I_{a}-I_{1}\right)\right. \\
& \left.-2 C_{\varepsilon} I_{a(\tau-1)} \zeta+D_{\zeta}\left(I_{T \tau}-I_{1}\right)_{\zeta}\right\}+\Delta U(\phi),
\end{aligned}
$$

where

$$
\left.\begin{array}{l}
A_{\zeta}=G\left\{1+y^{2} /(1-y)+K y^{3} /(1-y)^{2}\right\}, \\
B_{\zeta}=G\left\{y^{2} /(1-y)+K y^{4} /(1-y)^{2}\right\}, \\
C_{\zeta}=G y^{2} /(1-y), \\
D_{\zeta}=G y^{2}\left\{1+y^{2} /(1-y)\right\},
\end{array}\right\}
$$

and

$$
\begin{aligned}
& K=K_{o}(1-\zeta), \\
& \quad K_{o}=g_{f}{ }^{(m)} /\left(g_{r} d\right) .
\end{aligned}
$$

It is a notable fact that the deformed state of the system cannot be. determined only by $\Delta F(\alpha)$, but also by the following condition on the volume: of the system as shown in a Eq. (10);

$$
\phi=1+Y(\gamma-1)
$$


especially

$$
\phi_{\mathrm{IL}}=1+Y(\gamma-1) .
$$

$\left(4^{\circ}\right)$ Stability of Deformation and Determination of Cavity: The size of cavity is represented in term of $\gamma$ or $\phi_{\mathrm{II}}$ which is determined by the stability condition of the deformation in the ideal state II.

The determination equation of $\phi_{\mathrm{II}}$ is obtained by the minimization of $\Delta F_{\text {II }}(\boldsymbol{a})$ under the incidental condition in Eq. (10a).

Under the simple elongation $\alpha_{1}=\alpha_{2}=\beta, \alpha_{3}=\alpha, \Delta F_{\mathrm{II}}(\alpha)$ which is given by taking $\zeta=1$ in Eq. (25) and the incidental condition are written in these forms,

$$
\begin{aligned}
\Delta F_{\mathrm{II}}(\alpha)= & \Delta F_{r \Pi \mathrm{I}}(\alpha) \\
= & (1 / 2)\left\{A_{1}\left(\alpha^{2}+2 \beta_{\mathrm{II}}{ }^{2}-3\right)-2 \beta_{1}\left(\alpha+2 \beta_{\mathrm{II}}-3\right)\right. \\
& \left.\quad-2 C_{1} \alpha(\gamma-1)+D_{1}\left(\gamma^{2}-1\right)\right\}+\Delta U\left(\phi_{\mathrm{II}}\right)
\end{aligned}
$$

and

$$
\phi_{\mathrm{II}}=\alpha \beta^{2} \mathrm{II}=1+Y(\gamma-1) \text {. }
$$

The condition for the stability as $\alpha$ being kept constant is given by

$$
\left(\frac{\partial \Delta F_{\Pi}}{\partial \beta_{\mathrm{II}}}\right)_{a, r}=0, \quad\left(\frac{\partial \Delta F_{\mathrm{II}}}{\partial r}\right)_{a, \beta_{\mathrm{II}}}=0
$$

and it is written in the following formula:

$$
\frac{A_{1} \beta_{\text {II }}-B_{1}}{C_{1} \alpha-D_{1}}=\frac{\alpha \beta_{\text {II }}}{Y}
$$

or using Eq. (25a) and Eq. (26a), it becomes

$$
\begin{aligned}
\left(1-y+y^{2}\right) \sqrt{\alpha} \sqrt{\phi_{\mathbb{I}}^{3}}- & \left\{y^{3} \sqrt{\alpha^{3}}+\left(1-y+y^{2}\right)\left(1-y^{3}\right) \sqrt{\alpha}\right. \\
- & \left.y^{4}\left(1-y+y^{2}\right) / \sqrt{\alpha}\right\} \sqrt{\phi_{\mathbb{I I}}}-y^{6}=0 .
\end{aligned}
$$

From Eq. $\left(27^{\prime \prime}\right)$ the next formula is derived

$$
\phi_{I^{\prime}}(1)=y^{3} \frac{2\left(1+y-y^{2}\right)}{2\left(1-y+y^{2}\right)} \div y^{3} \frac{1+y-y^{2}}{1-y+y^{2}}
$$

and it is to be used in next section.

\section{TENSION AND YOUNG'S MODULUS}

$\left(1^{\circ}\right)$ Tension: In the simple elongation $\alpha_{1}=\alpha_{2}=\beta, \quad \alpha_{3}=\alpha$, the internal pressure $p=-\left(\partial \Delta U / \partial V^{\prime}\right)$ is determined by the fact

$$
\sigma_{1}=\partial \Delta F / \partial \alpha_{1}=0 \quad \text { or } \quad \sigma_{2}=\partial \Delta F / \partial \alpha_{2}=0 .
$$

Using this relation and Eq. (25), the tension $\sigma_{3}=\sigma$ becomes

$$
\sigma=(\partial \Delta F / \partial x)_{\beta}
$$




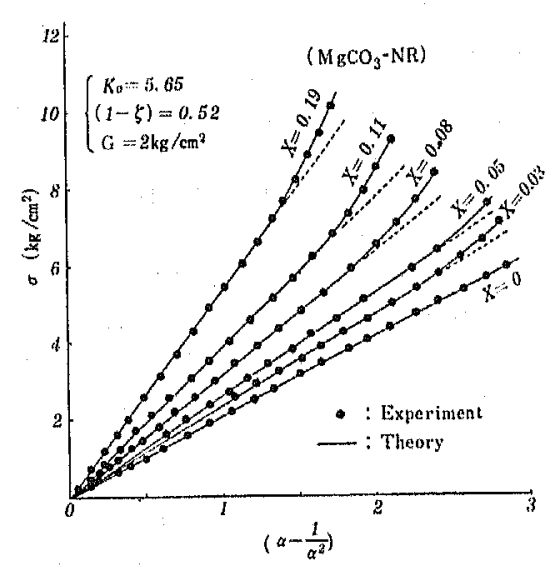

Fig. 6 An example for relation of tension versus elongation in filled rubber vulcanizates5). Theoretical curves are calculated from Eq. (28').

$$
=A_{\zeta}\left(\alpha-\phi / \alpha^{2}\right)-B_{\xi}\left(1-\sqrt{\phi / \alpha^{3}}\right)-\left(C_{\xi} / Y\right)(\phi-1),
$$

or using Eq. (25a)

$$
\begin{aligned}
\sigma=G[\{1 & \left.+\frac{y^{2}}{1-y}+K \frac{y^{3}}{(1-y)^{2}}\right\}\left(\alpha-\frac{\phi}{\alpha^{2}}\right) \\
& \left.-\left\{\frac{y^{2}}{1-y}+K \frac{y^{4}}{(1-y)^{2}}\right\}\left(1-\sqrt{\frac{\phi}{\alpha^{3}}}\right)-\frac{\phi-1}{y(1-y)}\right] .
\end{aligned}
$$

The tension $\sigma$ in Eq. $\left(28^{\prime}\right)$ is divided into three parts: the volume effect which contains the volume concentration function $y^{2} /(1-y)$, the surface effect which contains the surface concentration function $y^{3} /(1-y)^{2}$ and the factor $K$, and the cavitation effect $(\phi-1) /\{y(1-y)\}$ which is the product of the volume function $y^{2} /(1-y)$ and the size of the cavity $(\gamma-1) c=(\phi-1) / y^{3}$. When $y$ tends to 0 in Eq. $\left(28^{\prime}\right), \sigma$ agrees with

$$
\sigma=G\left(\alpha-1 / \alpha^{2}\right)
$$

in usual theory.

As one of the example the comparison between the theoretical tension and the experimental one ${ }^{5)}$ is shown in Fig. 6.

$\left(2^{\circ}\right)$ Initial Young's Modulus: To obtain Young's modulus $E$ at $\alpha=100 \%$, differentiating $\sigma$ in Eq. $\left(28^{\prime}\right)$ with respect to $\alpha, E$ can be calculated as follows:

$$
\begin{aligned}
E & =\left(\frac{\partial \sigma}{\partial \alpha}\right)_{a=1} \\
& =3\left(A_{\zeta}-B_{\zeta} / 2\right)\left\{1-\phi^{\prime}(1) / 3\right\}-\left(C_{\zeta} / Y\right) \phi^{\prime}(1),
\end{aligned}
$$

or using the Eq. (25a) and $\phi^{\prime}(1)=\zeta \phi_{\text {II }}{ }^{\prime}(1)$ in Eq. (10) 


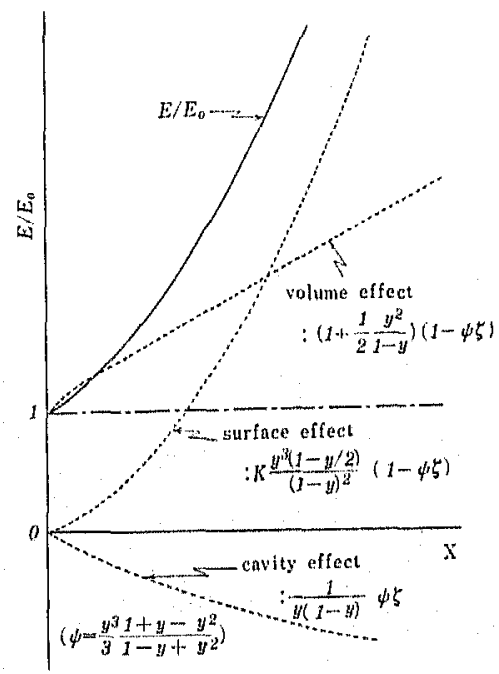

Fig. 7 Three effects and reinforcing tendency in Young's modulus.

$$
\begin{aligned}
E & =E_{0}\left[\left\{1+\frac{1}{2} \frac{y^{2}}{1-y}\right\}\left(1-\psi_{\zeta}^{\zeta}\right)\right. \\
& +K \frac{y^{3}(1-y / 2)}{(1-y)^{2}}\left(1-\psi_{\zeta}\right) \\
& \left.-\frac{y^{2}}{1-y}-\frac{\psi_{\zeta}}{y^{3}}\right]
\end{aligned}
$$

..........(volume effect) (surface effect) (cavitation effect)

where

$$
\begin{aligned}
\phi & =\phi_{I^{\prime}}(I) / 3 \\
& =\frac{y^{3} I+y-y^{2}}{3} \frac{1-y+y^{2}}{},
\end{aligned}
$$

and $E_{o}$ is the initial Young's modulus in the ordinary theory;

$$
E_{0}=3 G \text {. }
$$

It is clear that $E$ tends to $E_{o}$ in the ordinary theory as $y$ tends: to 0 .

As shown in Eq. (29), $E$ is also divided into three effect, and they are shown in Fig. 7 schematically.

The theoretical view of the reinforcement in Young's modulus $E$ with respect to several valus for $K_{0}$ and $(1-\zeta)$ is shown in Fig. 8. There are various types of the reinforcing curves which range from the linear property to the curved one corresponding to our experience. As examples of the linear property and curved one, the measured curves for precipitated calcium carbonate filled natural rubber vulcanizate and sillica filled one are shown in 

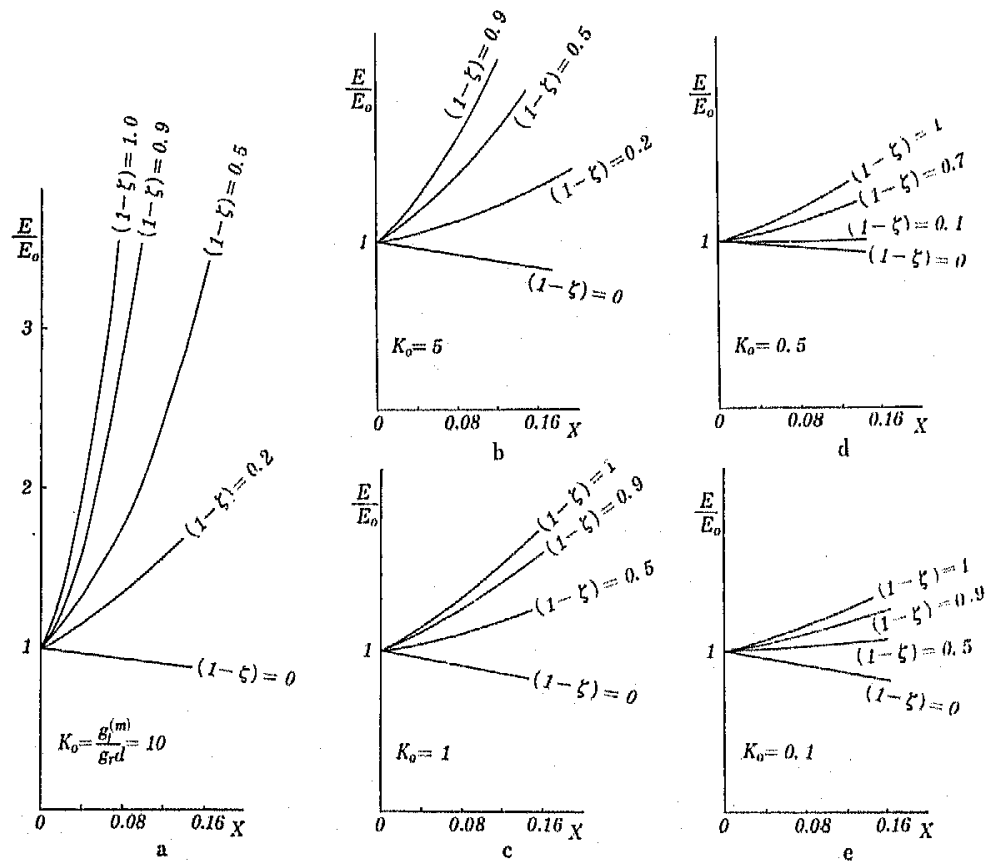

Fig. 8 Theoretical view of reinforcement in initial Young's modulus (calculated from Eq.(29)).

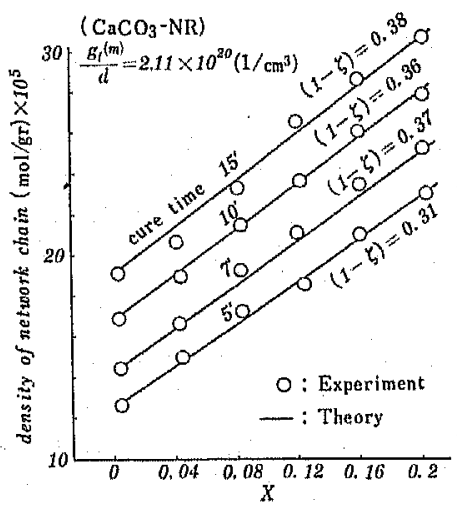

Fig.9 a An example of linear reinforcing property in modulus. 6) Theoretical curves are calculated from Eq.(29)

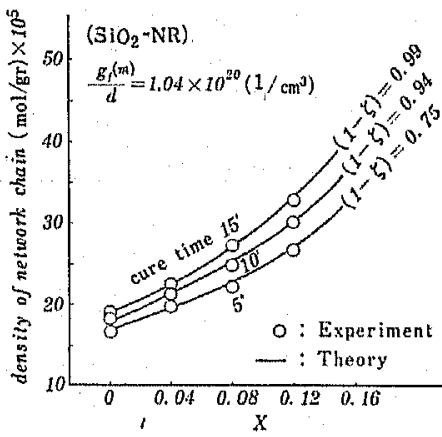

Fig.9 b An example of curved rein. forcing property in modulus. ${ }^{6}$ ) Theoretical curves are calculated from Eq. (29) 
Fig. 9, a and b, respectively. ${ }^{6}$ )

\section{THREE EFFECTS AND INTERNAL MECHANISM OF FILLER REINFORCEMENT}

Each quantity in the theory are divided into three parts which are the volume effect, the surface effect and the cavitation effect, as seen in $\sigma$ and $E$. In general the volume effect has the volume modulus $G=g_{r} k T$ and a concentration fnuction $y^{2} /(1-y)$, and the surface effect has the surface modulus $G^{\prime}=g_{j} k T / d$ (converted volume one) and a concentration function $y^{3} /(1-y)^{2}$.

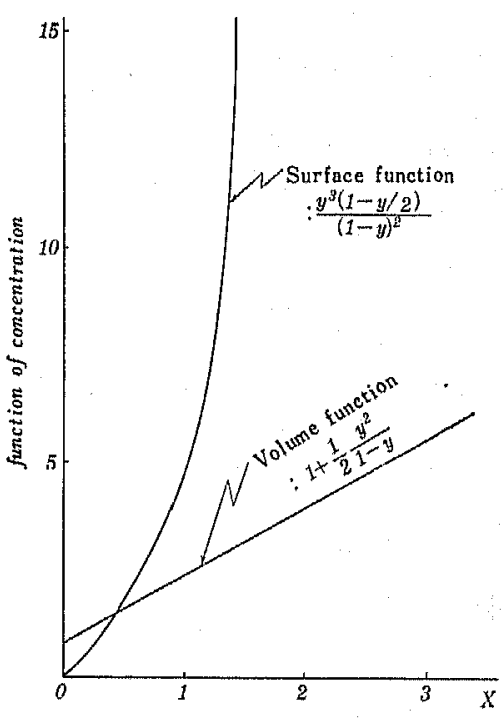

Fig. 10 Volume concentration function and surface concentration function in Young's modulus.
The concentration dependencies of filler reinforcement in the theory are determined through the volume concentration function $y^{2} /(1-y)$ due to the spatial distribution of the ordinary network chains within rubbery medium and the surface concentration function $y^{8} /(1-y)^{2}$ due to the surface distribution of the adhered one over the particles. Although the cavitation effect is influenced also through the volume function $y^{2} /(1-y)$, it's reason is understood that the cavitation effect due to the spatial distribution of the network chains in the cavity with volume density 0 .

The volume concentration function $y^{2} /(1-y)$ increases almost linearly with filler concentration $X$ in the wide range of $0<X<9$, (it is easily verified that $y^{2} /(1-y) \div 3 X$ for $\left.0<X<9\right)$, while the surface one $y^{3} /(1-y)^{2}$ increases very rapidly with increasing $X$, as shown in Fig. 10. Hence the linear property of the volume effect is clearer at the lower range of $X$, and the curved property of the surface

effect. comes to have relative merit at the higher range of $X$. The curveture in the reinforcing curves increases or decreases in response to the magnitude of $K=g_{f} /\left(g_{r} d\right), \quad\left(g_{f}=g_{f}^{(m)}(1-\zeta)\right)$ which is a ratio of the surface density $g_{f} / d$ (coverted volume one) to the volume density $g_{r}$ of the network chains. Therefore $K$ plays a role of an amplibication factor of the surface effect which is relative to the volume effect.

It may be clear from the above consideration that the reinforcing behaviour of the every filler (not only the inert filler but also the active filler) can be understood in the whole aspect.

For example, let us see again the theoretical view of the reinforcing curves of $E$ in Fig. 8. 


\section{SWELLING ELASTICITY AND CONDITION FOR SWELLING EQUILIBRIUM}

In the swelling of the filler-reinforced heterogeneous specimen, the distinction between the internal swelling and the apparent (external) swelling is necessary. It is impossible to distinguish without having a theory based upon the heterogeneous character of the filled specimen,

$\left(\mathbf{1}^{\circ}\right)$ The Tension and Young's Modulus in Swollen State: Let us consider the swollen deformed filled specimen in the equilibrium state with apparent deformation $\boldsymbol{\alpha}^{\prime}$ due to the solvent absorbed and the force externally applied.

Let us assume that the volumes of rubbery medium and solvent in the swollen deformed gel are additive. The volume of the specimen in the general adhesion state becomes

$$
V^{\prime}=V_{r}+M(4 \pi / 3) d^{3} \gamma \zeta_{s}+n V_{l},
$$

where $n_{l}$ is the number of moles of solvent with molar volume $V_{l}$ in the swollen medium and $\zeta_{s}$ denotes $\zeta$ in the swollen state. If the volume fraction of chain molecules within the swollen medium denotes $v_{2}$, the internal swelling ratio is given by $1 / v_{2}$ :

For simplicity the process from the natural state to the above state is divided into the next two stages:

(i) Isotropic swelling process from the natural state to the state of external swelling ratio $q^{3}$ :

$$
\mathbf{1} \longrightarrow \boldsymbol{\alpha}_{\mathrm{s}}=q \mathbf{1},
$$

where the suffix $s$ denotes the swollen quantities in general.

(ii) Deformation process without a volume change in the swollen medium

$$
\alpha_{s} \longrightarrow \alpha^{\prime}=\alpha \alpha_{s}=q \alpha,
$$

where $\boldsymbol{\alpha}$ is deformation due to the external applied forces.

In the first stage, the initial volume of specimen $V=M(4 \pi / 3) D^{\text {s }}$ is expanded to $V_{s}=q^{3} V=M(4 \pi / 3) D^{\prime 3}$, where $D^{\prime}=q D$. Then the concentration which sways the properties of the swollen specimen is not $y$, but $y^{\prime}$ defined as $d / D^{\prime}$, and

$$
\begin{aligned}
y^{\prime}=d / D^{\prime} & =(d / D)\left(D / D^{\prime}\right) \\
& =y / q(<y) .
\end{aligned}
$$

Therefore the deformation theorem of the network chains in the swollen double-network system is obtained thus

$$
\boldsymbol{r}^{\prime}=\boldsymbol{\alpha}_{p}\left(y^{\prime}\right) \boldsymbol{r}
$$

where $\boldsymbol{a}_{p}\left(y^{\prime}\right)$ is the local deformation in which $y$ in Eq. (4) is replaced by $y^{\prime}$.

The entropy part of deformation energy of the swollen system $\Delta \mathrm{F}_{e}\left(\boldsymbol{\alpha}^{\prime}\right)$ under the simple elongation is obtained in such a way, when the quantities $\alpha$, $\zeta$ and $y$ in Eq. (25) are substituted by $a^{\prime}, \zeta$ s and $y^{\prime}$ respectively, that 
where

$$
\begin{gathered}
\Delta F_{c}\left(\boldsymbol{\alpha}^{\prime}\right)=(1 / 2)\left[A_{\zeta^{\prime}}\left(\alpha^{\prime 2}+2 \beta^{\prime 2}-3\right)-2 B_{\zeta^{\prime}}\left(\alpha^{\prime}+2 \beta^{\prime}-3\right)\right. \\
\left.-2 C_{\xi^{\prime}} \alpha^{\prime}(\gamma-1) \zeta_{s}+D_{\zeta^{\prime}}\left(\gamma^{2}-1\right) \zeta_{s}\right\}
\end{gathered}
$$

$$
A_{\xi^{\prime}}=A_{\zeta}\left(y^{\prime}\right), \quad B_{\xi^{\prime}}=B_{\zeta}\left(y^{\prime}\right), \text { etc. }
$$

in Eq. (25a).

The changes of free energy $\Delta F_{1}$ and $\Delta F_{2}$ in the processes $1 \longrightarrow \boldsymbol{\alpha}_{s}$ and $\boldsymbol{\alpha}_{s}$ $\longrightarrow \boldsymbol{a}^{\prime}$ are calculated in the following ways,

(i) $1 \longrightarrow \alpha_{s}:$ (taking account of non-cavitation: $\gamma=1$ ):

$$
\begin{aligned}
\Delta F_{1} & =\left[\Delta F_{e}\left(y^{\prime}, \alpha_{s}\right)-\Delta F_{e}(y, 1)\right]_{\gamma=1} \\
& =(3 / 2)\left\{A_{\zeta^{\prime}}\left(q^{2}-1\right)-2 B_{\xi}(q-1)\right\} .
\end{aligned}
$$

(ii) $\alpha_{s} \longrightarrow \alpha^{\prime}=\alpha \alpha_{s}:\left(\right.$ take care that $\alpha^{\prime}=q \alpha, \beta^{\prime}=q \beta$ in Eq. (32))

$$
\begin{aligned}
\Delta F_{2}= & \Delta F_{e}\left(y^{\prime}, \alpha^{\prime}\right)-\Delta F_{1} \\
= & (1 / 2)\left[A_{\zeta^{\prime}}\left\{q^{2}\left(\alpha^{2}+2 \beta^{2}\right)-3\right\}-2 B_{\zeta}^{\prime}\{q(\alpha+2 \beta)-3\}\right. \\
& \left.-2 C_{\zeta^{\prime}} q \alpha(\gamma-1) \zeta_{s}+D_{\zeta^{\prime}}\left(\gamma^{2}-1\right) \zeta_{s}\right] \\
& -(1 / 2)\left\{A_{\zeta^{\prime}}\left(3 q^{2}-3\right)-2 B_{\zeta^{\prime}}(3 q-3)\right\} \\
= & (1 / 2)\left\{q^{2} A_{\zeta^{\prime}}\left(\alpha^{2}+2 \beta^{2}-3\right)-2 q B_{\zeta}(\alpha+2 \beta-3)\right. \\
& \left.-2 q C_{\zeta^{\prime}} \alpha(\gamma-1) \zeta_{s}+D_{\zeta}^{\prime}\left(\gamma^{2}-1\right) \zeta_{s}\right\} .
\end{aligned}
$$

Then the change of free energy $\Delta F_{s}(\alpha)$ per unit swollen volume in the whole process can be written thus,

$$
\begin{aligned}
\Delta F_{s}= & \left(V / V^{\prime}\right) \Delta F_{2}=q^{-3} \Delta F_{2} \\
= & \left(q^{-2} / 2\right)\left\{q A_{\zeta^{\prime}}\left(\alpha^{2}+2 \beta^{2}-3\right)-2 B_{\zeta^{\prime}}(\alpha+2 \beta-3)\right. \\
& \left.\quad-2 C_{\xi^{\prime}} \alpha(\gamma-1) \zeta_{s}+q^{-1} D_{\zeta^{\prime}}\left(\gamma^{2}-1\right) \zeta_{s}\right\}+\Delta U\left(\phi_{s}\right),
\end{aligned}
$$

where $\phi_{s}$ is the volume condition corresponding to the process (ii) defined as

$$
\begin{aligned}
\phi_{s}=V / V_{s} & =\alpha \beta^{2} \\
& =1+Y^{\prime}(\gamma-1) \zeta_{s} .
\end{aligned}
$$

From the above equations the tension $\sigma_{s}$ and Young's modulus $E_{s}$ in the swollen state are obtained in a similar way as in $\sigma$ and $E$;

and

$$
\begin{aligned}
\sigma_{s}=G q^{-2}[q & \left\{1+\frac{y^{\prime 2}}{1-y^{\prime}}+K_{s} \frac{y^{\prime 3}}{\left(1-y^{\prime}\right)^{2}}\right\}\left(\alpha-\frac{\phi_{s}}{\alpha_{2}}\right) \\
& -\left\{\frac{y^{\prime 2}}{1-y^{\prime}}+K_{s} \frac{y^{\prime 4}}{\left(1-y^{\prime}\right)^{2}}\right\}\left(1-\sqrt{\left.\frac{\phi_{s}}{\alpha^{3}}\right)-\frac{\phi_{s}-1}{y^{\prime}\left(1-y^{\prime}\right)}}\right]
\end{aligned}
$$

where

$$
\begin{aligned}
E_{s}=3 G & q^{-2}\left[\left\{q \left(1+\frac{y^{\prime 2}}{1-y^{\prime}}+K_{s} \frac{y^{\prime 2}}{\left(1-y^{\prime}\right)^{2}}\right.\right.\right. \\
& \left.\left.-\frac{1}{2}\left(\frac{y^{\prime 2}}{1-y^{\prime}}+K_{s} \frac{y^{\prime}}{\left(1-y^{\prime}\right)^{2}}\right)\right\}\left(1-\psi_{s} \zeta\right)-\frac{\psi_{s} \zeta_{s}}{y^{\prime}\left(1-y^{\prime}\right)}\right]
\end{aligned}
$$




$$
K_{s}=K_{0}\left(1-\zeta_{s}\right) ; \quad\left(K_{0}=g_{f}^{(m)} /\left(g_{r} d\right)\right)
$$

and

$$
\psi_{s}=\frac{y^{\prime 3}}{3} \frac{\left(-q^{-1}+q y^{\prime}\right)\left(1-y^{\prime}+y^{\prime 2}\right)+3}{\left\{q^{-1}\left(2+y^{\prime 3}\right)+q y^{\prime 4}\right\}\left(1-y^{\prime}+y^{\prime 2}\right)-y^{\prime 3}} .
$$

As a matter of course if the concentration $y=y^{\prime} q$ tends to $0, \sigma s$ and $E_{s}$ become $\sigma$ and $E$ respectively in the ordinary theory;

$$
\sigma_{s} \longrightarrow \sigma=G v_{2}^{1 / 3}\left(\alpha-1 / \alpha^{2}\right), \quad(\text { as } y \longrightarrow 0)
$$

and

$$
E_{s} \longrightarrow E=E_{0} v_{2}^{1 / 3}, \quad(\text { as } y \rightarrow 0)
$$
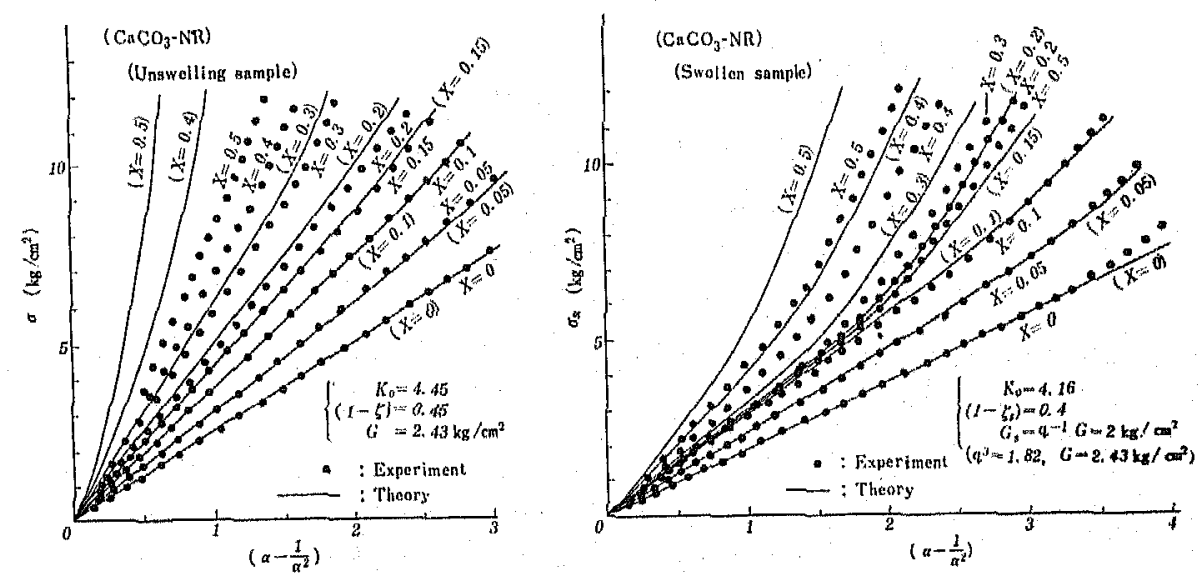

Fig. 11 Comparison between tension $\sigma$ and swelling tension 3 ). Theoretical curves are calculated from Eqs.(28') and (37) respectively.

The comparison between the theory and the experiment ${ }^{3}$ ) is shown in Fig. 11 for $\sigma$ and os over a wider range $(0 \leqq X \leqq 0.5)$ than one in Fig. 6 . For the specimens of higher concentration $X=0.4,0.5$, the experimental curves lie far under the theoretical ones. It can be understood that the treatment of $(1-\zeta)=$ constant (Fig. 5 cf.) adopting here becomes unsuitable at such a high concentration range, because of the excess of local deformation. The theoretical view of the swelling effect on Young's modulus $E$ is shown in Fig. 12.

$\left(2^{\circ}\right)$ The Equilibrium Condition in Free Swelling : Let the specimen be immersed in the solvent, and it is invaded by the liquid molecules until the initial volume $V$ increases to the equilibrium one $V_{s}=q^{3} V$ without an external force applied in the isothermal process. 


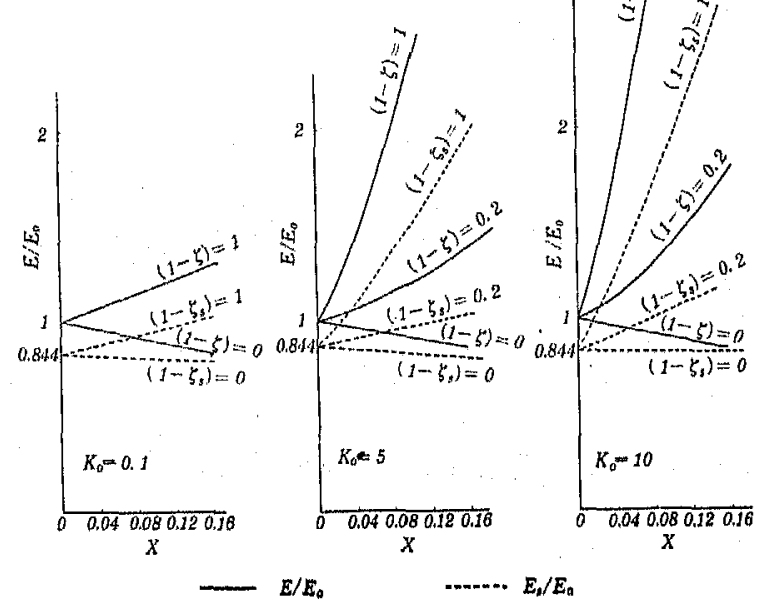

Fig.12 Comparison between initial Young's modulus $E$ in unswelling state and $\Gamma_{s}$ in swollen state. (calculated from Eqs. (29) and (38))

From the equations (30) and (33), the swelling ratio $q^{3}$ and the energy of deformation $\Delta F_{e}$ of the system in this case can be written as follows,

$$
q^{8}=1+n_{l} V_{l} / V
$$

and

$$
\Delta F_{e}=(3 / 2) V\left\{A_{\zeta^{\prime}}\left(q^{2}-1\right)-2 B_{\zeta}{ }^{\prime}(q-1)\right\} .
$$

Using these equations, we get

$$
\begin{aligned}
\frac{\partial \Delta F_{e}}{\partial n_{l}} & =\frac{\partial \Delta F_{e}}{\partial q}-\frac{\partial q}{\partial n_{l}} \\
& =V_{l} q^{-2}\left(A_{\zeta}^{\prime} q-B_{\zeta}^{\prime}\right) .
\end{aligned}
$$

As well known the invasion of the liquid molecules into the rubbery medium owes to the decreasing nature in the free energy of mixing $\Delta F_{m}$, while the invasion of the liquid brings about the increase of the elastic energy $\Delta F_{c}$ stored in extending double network system due to swelling. The condition for equilibrium with respect to the invasion of liquid is that, for a small variation $\delta n_{l}$ moles of the liquid content, the decrease $\left(\partial \Delta F_{m} / \partial n_{l}\right) \delta n_{l}$ in $\Delta F_{m}$ is canceled with the increament $\left(\partial \Delta F_{e} / \partial n_{l}\right) \partial n_{l}$ in $\Delta F_{e}$. In other words the free energy of the system $\Delta F=\Delta F_{n}+\Delta F_{a}$ is minimized with respect to $n_{l}$ :

$$
\frac{\partial \Delta F}{\partial n_{l}}=\frac{\partial \Delta F_{m}}{\partial n_{l}}+\frac{\partial \Delta F_{e}}{\partial n_{l}}=0
$$



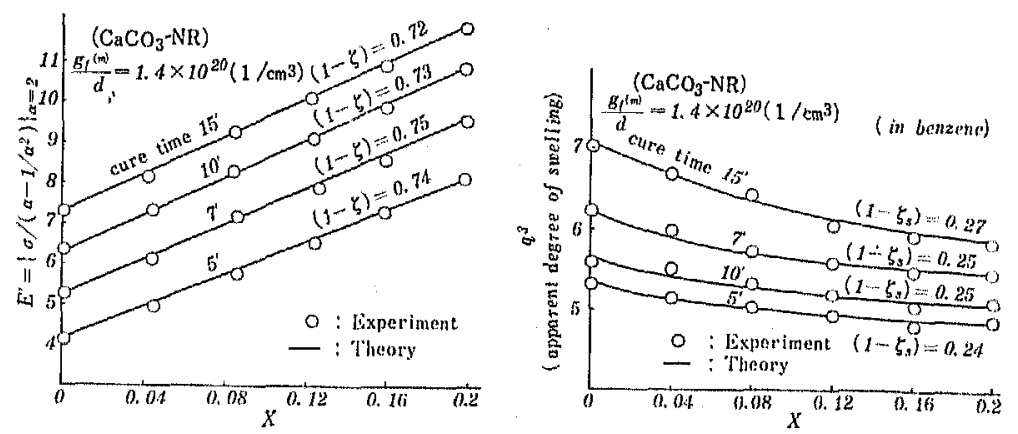

Fig. 13 An example of reinforcement in unswelling Young's modulus and resistance to swelling in benzene. ${ }^{6)}$ Theoretical curves are calculated fron Eqs. $\left(28^{\prime}\right)$ and (43)

Using Eq. (40) and the Flory-Huggins expression for $\partial \Delta F_{m} / \partial n_{l}$ such that

$$
\frac{\partial \Delta F_{m}}{\partial m_{\ell}}=R T\left\{\log \left(1-v_{2}\right)+v_{2}+\mu v_{2}^{2}\right\}
$$

the equilibrium condition in Eq. (41) becomes

$$
\begin{aligned}
\log \left(1-v_{2}\right) & +v_{2}+\mu v_{2}{ }^{2}+\frac{V l G}{R T} q^{2}\left[q \left\{1+\frac{y^{\prime 2}}{1-y^{\prime 2}}\right.\right. \\
& \left.\left.+K_{s} \frac{y^{\prime 3}}{\left(1-y^{\prime}\right)^{2}}\right\}-\left\{\frac{y^{\prime 2}}{1-y^{\prime}}+K_{s} \frac{y^{\prime 4}}{\left(1-y^{\prime}\right)^{2}}\right\}\right]=0
\end{aligned}
$$

where $\mu$ is the Huggins coefficient.

As an example, the modulus $E^{\prime}=\left\{\sigma /\left(\alpha-1 / \alpha^{2}\right)\right\}_{a=2}$ in the unswollen state and swelling ratio $q^{3}$ are compared with those calculated from Eq. $\left(28^{\prime}\right)$ and Eq. (43) as shown in Fig. 13. The same specimens are used in both experiments. ${ }^{6)}$

\section{CONSIDERATION OF GENERALIZED SURFACE ENERGY AND SURFACE LAYER}

$\left(1^{\circ}\right)$ The Theory Taking Account of the Surface Energies besides $\Delta F_{f}$ : When the surface of the cavity is extending, several kinds of surface energies induced by the various molecular mechanisms in addition to $\Delta F_{f}$, may appear, if we consider in detail.

Although such energies cannot be estimated similarly as the energy of the external surface in the ordinary theory of rubber elasticity, the phenomenological treatment of the surface energies of cavity is not always difficult in the present theory.

In general, the surface energy of any kind is proportional to the surface area in question and the every surface force is a function of that area.

In the present theory the total surface area of the cavity $S(\gamma)$ under the external deformation $\boldsymbol{a}$ is written as 


$$
S(\gamma)=M 2 \pi d^{2}\left\{1+\left(\gamma / \sqrt{1-1 / r^{2}}\right) \sin ^{-1} \sqrt{1-1 / \gamma^{2}}\right\}
$$

then the resultant surface force $\Gamma(\gamma)$ based on the various energies is a function of $\gamma$. Therefore, the work $H(\gamma)$ done during the extension of the surface from $S(1)$ to $S(r)$ becomes

$$
H(\gamma)=\int_{1}^{\gamma} \Gamma(\gamma) d S(\gamma)
$$

and it must be added to the $\Delta F(\boldsymbol{\alpha})$ in Eq. (25). Let $\Delta F^{*}(\boldsymbol{\alpha})$ be the energy of deformation of the system in this case and it is given by

$$
\begin{aligned}
\Delta F^{*}(\alpha)= & \Delta F+H(\gamma) \\
= & (1 / 2)\left\{A_{\zeta}\left(\alpha^{2}+2 \beta^{2}-3\right)-2 B_{\zeta}(\alpha+2 \beta-3)\right. \\
& \left.-2 C_{\zeta} \alpha(\gamma-1) \zeta+D_{\zeta}\left(\gamma^{2}-1\right) \zeta\right\}+\Delta U(\phi)+H(\gamma) .
\end{aligned}
$$

From this $\Delta F^{*}(\alpha)$ and the condition on the volume in Eq. (10) the tension $\sigma^{*}$ in this case is obtained in the same way as before,

$$
\begin{aligned}
\sigma^{*}=G[ & {\left[1+\frac{y^{2}}{1-y}+K \frac{y^{3}}{(1-y)^{2}}\right\}\left(\alpha-\frac{\phi}{\alpha^{2}}\right) } \\
& \left.-\left\{\frac{y^{2}}{1-y}+K \frac{y^{4}}{(1-y)^{2}}\right\}\left(1-\sqrt{\frac{\phi}{\alpha^{3}}}\right)-\frac{\phi-1}{y(1-y)}\right] .
\end{aligned}
$$

Although $\sigma^{*}$ does not differ from the previous tension $\sigma$ in Eq. (28') formally, but the determinate equation for $\gamma$ namely

$$
\frac{A_{\zeta} \beta_{\mathrm{II}}-B_{\zeta}}{C_{\zeta} \alpha-D_{\zeta \gamma}-\frac{\partial H}{\partial \gamma}}=\frac{\alpha \beta_{\mathrm{II}}}{Y}
$$

differs from the previous one in Eq. $\left(27^{\prime}\right)$ only a term $\partial H / \partial \gamma$.

$\left(2^{\circ}\right)$ On the Surface Layer Coverling the Particles: The state and the structure of the boundary of filler to rubbery medium is not clear at present. However, many investigators believe that there are perhaps some clegenerated layers consisting of rubber vulcanizates around the surface of the filler with finite thickness.

Such a layer surrounding a dispersed particle may be described in terms of some simplifing picture, e.g., the following picture is considered : the overcuring layer enclosing each $d$-sphere with the thickness $\delta$ and the volume density $g_{l}(R)$ around the position $R$. Perhaps $g_{l}(R)$ may be a decreasing continuous function at $d \leqq R<d+\delta$, having a limiting value of $\lim _{R \rightarrow d+\delta} g_{l}(R)=g_{r}$.

In this case, the analysis requires increases in the number of observables, e. g., not only $g_{l}(R)$ but also $\delta$ appears as additional parameter in this picture.

At least if one of those informations is inacurrate, the theory based upon such a model falls into a mere speculation because of the excess in parameters. Actually the thickness of the boundary layer may not be defined without the precise data.

Such being the case, let us avoid the detailed discussion concerned with the structure and the state of the boundary layer at present. 
Neverthless, if the dispersion structure within the specimen in quesion is suitable to a picture with $\delta$ and $g_{l}(R)$ above mentioned, the present theory may remain valid, more or less, according to the actural state, by using of the converted surface density:

$$
\bar{g}_{f}=\frac{1}{d^{\prime 2}} \int_{d}^{d+\grave{o}} g_{l}(R) R^{2} d R
$$

where $d^{\prime}$ is a suitable parameter having some value in the range $d<d^{\prime}<d+i$. But the above method is not so desirable.

\section{APPENDIX}

A. Deformation Theorem of Network Chains: The definition of the internal deformation in Eq. (3) is written again

$$
R^{\prime}=a_{R} R,
$$

where

$$
\boldsymbol{\alpha}_{R}=\boldsymbol{\alpha}^{\prime}-(D / R) \boldsymbol{\alpha}^{\prime \prime}
$$

and

$$
\boldsymbol{\alpha}^{\prime}=(\boldsymbol{\alpha}-y \boldsymbol{\alpha}) /(1-y), \quad \boldsymbol{\alpha}^{\prime \prime}=\{y /(1-y)\}(\boldsymbol{\alpha}-\boldsymbol{\gamma}) .
$$

Taking the increment $\Delta R$ in $R$ about the point $\mathrm{P}$, the equation (A1) becomes

$$
\begin{aligned}
\Delta \boldsymbol{R}^{\prime} & =\Delta\left(\boldsymbol{\alpha}_{R} R\right) \\
& =\boldsymbol{\alpha}_{R}(\Delta R)+\left(\Delta \alpha_{R}\right) \boldsymbol{R} \\
& =\boldsymbol{\alpha}_{R}(\Delta R)+\left\{d\left(\alpha_{R}\right) / d R\right\} \Delta R \boldsymbol{R} \\
& =\boldsymbol{\alpha}_{R}(\Delta R)+\left(D / R^{2}\right) \boldsymbol{\alpha}^{\prime \prime}(\Delta R R) .
\end{aligned}
$$

As seen in Fig. 4, the following relations are clear

$$
R=R l, \quad \Delta R=\Delta R \cdot l \text {. }
$$

According to the above relations the $i$-component $\Delta R X_{i}$ of a factor $J R R$ in Eq. (A4) can be written as the following form

$$
\begin{aligned}
\Delta R X_{i} & =\left(\sum_{i=1}^{3} \Delta X_{j} l_{j}\right)\left(l_{i} R\right) \\
& =R \sum_{j=1}^{3}\left(l_{i} l_{j}\right) \Delta X_{j}
\end{aligned}
$$

or in the vector form

$$
\Delta R R=R(l l) \Delta R \text {. }
$$

Then the factor $\Delta R R$ in Eq. (A4) is replaced by this relation, the local deformation $\boldsymbol{\alpha}_{P}$ and the deformation theorem are so obtained that

$$
\begin{aligned}
\Delta \boldsymbol{R}^{\prime} & =\boldsymbol{\alpha}_{R} \Delta \boldsymbol{R}+(D / R) \boldsymbol{\alpha}^{\prime \prime}(l l) \Delta \boldsymbol{R} \\
& =\left\{\boldsymbol{\alpha}_{R}+(D / R) \boldsymbol{\alpha}^{\prime \prime}(l l)\right\} \Delta \boldsymbol{R},
\end{aligned}
$$




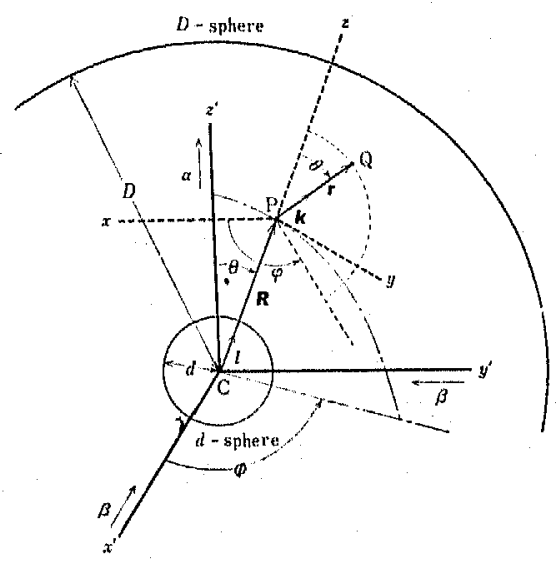

Fig. 14 Illustration of spherical coordinate systems $(R, \Theta, \Phi)$ and $(r, \theta, \varphi)$.

can $^{8}$ be written,

$$
d R=R^{2} \sin \Theta d \Theta d \Phi
$$

and

$$
d r=r^{2} \sin \theta d \theta d \varphi \text {. }
$$

Remember the definition of the probability $f(r, N) d r$ in the section $\mathbf{3}$ and since the network chains in the system are Gaussian and have the isotropic distribution with respect to $r, f(r, N) d r$ can be written such that

$$
f(r, N) d r=f_{N} w_{N}(r) r^{2} \sin \theta d r d \theta d \varphi
$$

where

$$
\begin{aligned}
w_{N}(r) & =\left\{3 /\left(2 \pi N a^{2}\right)\right\}^{3 / 2} \exp \left[-3 r^{2} /\left(2 N a^{2}\right)\right\}, \\
f_{N} & =g_{N} / g_{r}, \quad \text { (Eq. (16) cf. ). }
\end{aligned}
$$

The next relation is necessary in our calculation;

$$
\begin{aligned}
& \int\left(\boldsymbol{a}_{P} \boldsymbol{r} \cdot \boldsymbol{\alpha}_{P} \boldsymbol{r}\right) f(\boldsymbol{r}, N) d \boldsymbol{r} \\
& =\operatorname{Tr} .\left(\boldsymbol{a}_{P} \boldsymbol{a}_{P}\right) \int(\boldsymbol{r} \boldsymbol{r}) f(\boldsymbol{r}, N) d \boldsymbol{r}=\left(N a^{2} / 3\right) f_{N} \operatorname{Tr} .\left(\boldsymbol{\alpha}_{P} \boldsymbol{a}_{f}\right) .
\end{aligned}
$$

To prove this relation, let $k$ be the direction cosine of $r$, the next calculation is performed such that

$$
\begin{aligned}
& \int\left(\boldsymbol{\alpha}_{P} \boldsymbol{r} \cdot \boldsymbol{a}_{P} r\right) f(\boldsymbol{r}, N) d \boldsymbol{r}=\int \operatorname{Tr} .\left(\boldsymbol{\alpha}_{P} \boldsymbol{\alpha}_{P}\right)(\boldsymbol{r} \boldsymbol{r}) f(\boldsymbol{r}, N) d \boldsymbol{r} \\
& =\operatorname{Tr} .\left(\boldsymbol{\alpha}_{P} \boldsymbol{\alpha}_{P}\right) \int(\boldsymbol{r} \boldsymbol{r}) f(\boldsymbol{r}, N) d \boldsymbol{r}
\end{aligned}
$$




$$
\begin{aligned}
& =\operatorname{Tr} .\left(\boldsymbol{a}_{p}, \boldsymbol{a}_{p}\right) f_{N}\left\{\int_{0}^{\infty} r^{\mathrm{2}} w_{N}(r) 4 \pi r^{2} d r\right\}\left\{(1 / 4 \pi) \int_{0}^{2 \pi} \int_{0}^{\pi}(k k) \sin (l d(1) d \varphi\}\right. \\
& =f_{N}\left(N a^{2} / 3\right) \operatorname{Tr} .\left(\boldsymbol{\alpha}_{p} \boldsymbol{\alpha}_{p}\right),
\end{aligned}
$$

but the well known relation

$$
\int_{0}^{\infty}\left(r^{2}\right) w_{N}(r) 4 \pi r^{2} d r=N a^{2}
$$

and the relation

$$
\begin{aligned}
& \frac{1}{4 \pi} \int_{0}^{2 \pi} \int_{0}^{\pi}(k k) \sin 0 d \theta d \varphi \\
& =\frac{1}{4 \pi} \int_{0}^{\pi} \sin \theta d \theta \int_{0}^{2 \pi}\left(\begin{array}{llll}
\sin ^{2} \theta \cos ^{2} \varphi & \sin ^{2} \theta \sin \varphi \cos \varphi & \sin \theta \cos \theta \cos \varphi \\
\sin ^{2} \theta \cos \varphi \sin \varphi & \sin ^{2} \theta \sin ^{2} \varphi & \sin \theta \cos \theta \sin \varphi \\
\sin \theta \cos \theta \cos \varphi & \sin \theta \cos \theta \sin \varphi & \cos ^{2} \theta
\end{array}\right) d \varphi \\
& =\frac{1}{4 \pi} \int_{0}^{\pi}\left(\begin{array}{ccc}
\pi \sin ^{2} \theta & 0 & 0 \\
0 & \pi \sin ^{2} \theta & 0 \\
0 & 0 & 2 \pi \cos ^{2} \theta
\end{array}\right) \sin \theta d \theta \\
& =\frac{1}{3}\left(\begin{array}{lll}
1 & 0 & 0 \\
0 & 1 & 0 \\
0 & 0 & 1
\end{array}\right)
\end{aligned}
$$

are used in the above calculation.

In this connection, the following relation is equivalent to Eq. (B8)

$$
\int(l l) d \Omega=(4 \pi / 3) \mathbf{1},
$$

where $d Q$ is the solid angle such as $d Q=\sin \epsilon d \Theta d \Phi$.

By using of the equations (B6), (A7), (A2), (A3), and (B9), now the entropy part of the volume energy $F_{r}(\alpha)$ of the system per unit initial volume in Eq. (17) can be calculated in the following way,

$$
\begin{aligned}
F_{r}(\boldsymbol{\alpha})= & (M / V) \int g_{r} d \boldsymbol{R} \sum_{N}\left\{3 k T /\left(2 N a^{2}\right)\right\} \int \operatorname{Tr} .\left(\boldsymbol{\alpha}_{P} \boldsymbol{\alpha}_{P}\right)(\boldsymbol{r} \boldsymbol{r}) f(\boldsymbol{r}, N) d \boldsymbol{r} \\
= & (M / V) g_{r} \int \operatorname{Tr} .\left(\boldsymbol{\alpha}_{P} \boldsymbol{\alpha}_{P}\right) d \boldsymbol{R}\left[\sum_{N} f_{N}\left\{3 k T /\left(2 N a^{2}\right)\right\}\left(N a^{2} / 3\right)\right] \\
= & \left\{M g_{r} k T /(2 V)\right\} \int \operatorname{Tr}\left(\boldsymbol{\alpha}_{P} \boldsymbol{\alpha}_{P}\right) d \boldsymbol{R} \\
= & \{M G /(2 V)\} \operatorname{Tr} \cdot \int_{d}^{D} R^{2} d R \int\left[\boldsymbol{\alpha}^{\prime} \boldsymbol{\alpha}^{\prime}+2(D / R)\left\{\boldsymbol{\alpha}^{\prime} \boldsymbol{\alpha}^{\prime}(l l)-\boldsymbol{\alpha}^{\prime} \boldsymbol{\alpha}^{\prime \prime}\right\}\right. \\
& \left.+\left(D^{2} / R^{2}\right)\left\{(l l) \boldsymbol{\alpha}^{\prime \prime} \boldsymbol{\alpha}^{\prime \prime}(l l)-2 \boldsymbol{\alpha}^{\prime \prime} \boldsymbol{\alpha}^{\prime \prime}(l \boldsymbol{l})+\boldsymbol{\alpha}^{\prime \prime} \boldsymbol{\alpha}^{\prime}\right\}\right] d \Omega
\end{aligned}
$$




$$
\begin{aligned}
& =\{M G /(2 V)\}(4 \pi / 3) \operatorname{Tr} \cdot \int_{d}^{D} R^{2}\left\{3 \boldsymbol{\alpha}^{\prime 2}-4(D / R) \boldsymbol{\alpha}^{\prime} \boldsymbol{\alpha}^{\prime \prime}+2\left(D^{2} / R^{2}\right) \boldsymbol{\alpha}^{\prime \prime 2}\right\} d R \\
& =\left\{M 4 \pi D^{3} /(2 V)\right\}(G / 2) \operatorname{Tr} \cdot\left\{\left(1-y^{3}\right) \boldsymbol{\alpha}^{\prime 2}-2\left(1-y^{2}\right) \boldsymbol{\alpha}^{\prime} \boldsymbol{\alpha}^{\prime \prime}+2(1-y) \boldsymbol{\alpha}^{\prime \prime 2}\right\} \\
& =\{G / 2(1-y)\}\left\{\left(1-y+y^{2}\right) I_{\alpha \alpha}-2 y^{2} I_{a r}+y^{2}\left(1-y+y^{2}\right) I_{r r}\right\}, \quad \text { (B10) }
\end{aligned}
$$

but the relations $V=M(4 \pi / 3) D^{3}, G=g_{r} k T$ and $d / D=y$ are used in the midst of the above calculation.

Taking $r=1$ or $r=r$ in Eq. (B10), $\Delta F_{r I}(\alpha)$ or $\Delta F_{r I}(\alpha)$ is obtained at once.

C. On the Calculation of Surface Energy: Similarly the surface energy $F_{f I}(\alpha)$ of the system per unit initial volume in the ideal state $I$ is calculated. (Remember that $\Delta F_{f \mathbb{I}}(\alpha)=0$ ). Taking $r=1$ and using $d / D=y$ in Eq. (A7), the local deformation at the surface of $d$-sphere in the ideal state $I$ is reduced such that

$$
\left[\alpha_{P}\right]_{R=1}=1+y^{-1} \alpha^{\prime \prime}(l l)
$$

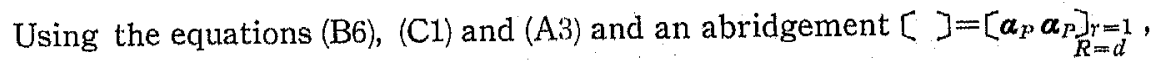
$F_{f \mathrm{~T}}(\boldsymbol{\alpha})$ can be calculated as

$$
\begin{aligned}
& F_{f_{\mathrm{I}}}(\boldsymbol{\alpha})=(M / V) \int d S \sum_{N}\left[3 k T /\left(2 N a^{2}\right)\right\} \int \operatorname{Tr} .[\quad](\boldsymbol{r} r) g_{f}(m) f(\boldsymbol{r}, N) d \boldsymbol{r} \\
& =(M / V)\left(g_{f}^{(m)} k T / 2\right) \int d^{2} d s \alpha \operatorname{Tr}[] \\
& =\left\{M d^{2} g_{f}{ }^{(m)} k T /(2 V)\right\} \operatorname{Tr} . \int\left[1+2 y^{-1} \boldsymbol{a}^{\prime \prime}(\boldsymbol{l} l)+y^{-2} \boldsymbol{\alpha}^{\prime \prime} \boldsymbol{\alpha}^{\prime \prime}(\boldsymbol{l} l)\right] d \Omega \\
& =\left\{M 4 \pi d^{2} / V\right\}\left(g_{f}(n) k T / 2\right)(1 / 3) \operatorname{Tr} .\left[31+2 y^{-1} \boldsymbol{\alpha}^{\prime \prime}+y^{-2-2} \boldsymbol{\alpha}^{1 / 2}\right] \\
& =\frac{g_{f}^{(m)} k T}{2 d} y^{\mathrm{3}}\left\{3 I_{1}+\frac{2}{1-y}\left(I_{a}-I_{1}\right)+\frac{1}{(1-y)^{2}}\left(I_{a a}-2 I_{a}-I_{1}\right)\right\} \text {. }
\end{aligned}
$$

It is notable that the specific surface area of the filler per unit volume of the specimen $M 4 \pi d^{2} / V=s$ appeares at the midst of Eq. (C2), and it is so rewritten that

$$
\begin{aligned}
s & =M 4 \pi d^{2} / V \\
& =(3 / d) y^{3},
\end{aligned}
$$

because of $V=M(4 \pi / 3) D^{3}$. According to Eq. (C2), the surface effect in the filler reinforcement is proportional to the specific ares $s$ or inversely propor * tional to the mean diameter $d$ under the constant concentration. 


\section{REFLRENCES}

1) e.g.

Smallwood, H. M. : J. Appl. Phys. 15, 758 (1944)

Guth, E.: Ibicl., 16, 20 (1945)

Rehenr, J. Jr.: J. Polymer Sci., 7, 519 (1951)

Blanchard, A.F., Parkinson, D.: Ind. Eng. Chem., 44, 799 (1952)

Bueche, A. M.: J. Polymer Sci., 25, 139 (1957)

Zapp, R. L., Guth, E.: Ind, Eng. chem., 43, 430 (1951)

Bueche, F.: J. Appl. Polymer Sci, , 4, 107 (1960); Ibid., 5, 271 (1961)

Mullins, L., Tobin, N. R.: Proc. Third Rubber Technol. Conf., 379 (1954) (London)

2) i) a general view of this theory:

etc.

Sato, Y.: High Polymer, 7, 396 (1958): Ibid. 11, 226 (1962) (in Japan): J. Japan Soci. Testing Materials 7, 290 (1.958): J. Soci. Rubber Ind. Japan, 31, 677 (1958): Gomu, 5, 32 (1962) (in Japan): Bonded Materials, 1, (5), 60 (1962): Ibid, . 1, (6), 35 (1962): Ibicl., 1, (7), 27 : Ibic., 1, (8) 22 (1962): Ibid 1, (9) 24 (1962) (1962) (in Japan) ii) original paper:

Sato, Y., Furukawa, J. : Busseiron Kenkyu, 2-2, 871 (1957): Ibid., 2-3, 1 (1958): Ibid., 2-3, 12 (1958): Ibid. 2-3, 274 (1958): Ibid., 2-3, 294 (1958): Ibic., 2-3, 404 (1958): Ibid., 2-3, 422 (1958): Ibid., 2-6, 9 (1959): Ibid., 2-6, 21 (1959): Ibid., 2-6, 29 (1959) (in Japan)

3) Sato, Y., Sekiguchi, N., Furukawa, J., Yamashita, S.: Busseiron Kenkyu, 2-6, 276 (1959) (in Japan)

4) Sato, Y., Sekiguchi, N., Furukawa, J.: Report on Progress in Polymer Physics in Japan, II, 73 (1959): Ibid, III, 76 (1960): Ibid., IV, 7 (1961): Ibid., V, 155 (1962)

5) Furukawa, J.: Chem. High Polymer, 6, 265 (1949)

Nishida, M., Furukawa, J. : Ibid., 6, 276 (1949) (in Japan)

6) Furukawa, J., Yamashita, S., Ito, M.; J. Soci. Rubber Ind. Japan, 30, 665 (1957)

Furukawa, J., Yamashita, S, Hida, Y.: Ibid, , 30, 673 (1957) 